\title{
AProAp - Um ambiente para projetos de aprendizagem
}

\author{
Marlon Ferrari' ${ }^{1}$, Orivaldo de Lira Tavares ${ }^{1}$, Crediné Silva de Menezes ${ }^{1,2}$ \\ ${ }^{1}$ Programa de Pós-Graduação em Informática - Universidade Federal do Espírito Santo (UFES) \\ Av. Fernando Ferrari, 514, Goiabeiras, CEP 29075-910, Vitória - ES - Brasil \\ ${ }^{2}$ Faculdade de Educação - Universidade Federal do Rio Grande do Sul (UFRGS) \\ Av. Paulo Gama, s/nº - Prédio 12201 - 90040-060 - Porto Alegre - RS - Brasil \\ mfmarlonferrari@gmail.com, \{tavares, credine\}@inf.ufes.br
}

\begin{abstract}
There are, currently, several telematic environments that support the development of learning projects. However, a detailed study reveals that these environments fail to provide the appropriate tools to assist all the steps that constitute a learning project, besides using tools that are poorly integrated and external to the environment. This paper presents an environment to support project-based learning AProAp, an environment with tools that address all development stages of a projectbased learning, in order to overcome the shortcomings of the current environments.

Resumo. Existem alguns ambientes telemáticos de apoio ao desenvolvimento de projetos de aprendizagem. Entretanto, um estudo minucioso revela que esses ambientes falham em oferecer as ferramentas apropriadas para cobrir todas as etapas que constituem um projeto de aprendizagem, além de usarem ferramentas pouco integradas e externas ao ambiente. Neste artigo é proposto um ambiente para suporte a projetos de aprendizagem - AProAp, um ambiente com ferramentas que contemplam a todas as etapas de desenvolvimento de um projeto de aprendizagem, de modo a superar as deficiências dos ambientes atuais.
\end{abstract}

\section{Introdução}

Um projeto de aprendizagem (PA) é uma arquitetura pedagógica [Carvalho et al., 2005], composta de um objetivo pedagógico (o que queremos aprender), estratégias pedagógicas (como iremos aprender) e recursos tecnológicos para a realização das estratégias. Durante todas as etapas do desenvolvimento de um projeto de aprendizagem, há a criação de conteúdos que permitem a construção de uma rede de conhecimento relacionada a cada questão de investigação [Fagundes et al., 2006].

No ambiente escolar, o grau de formalismo envolvido afeta a autonomia dos aprendizes e coloca a figura do professor como fundamentalmente envolvida e responsável por todos os processos de ensino-aprendizagem. Temos, portanto, que a classificação de um ambiente de aprendizagem, dentre outros critérios, segue uma linha entre sistematização e autonomia dos aprendizes. A sistematização define etapas e valida o processo de aprendizagem, formada de 
avaliações, exercícios, certificações e contratos entre os envolvidos no processo de aprendizagem. A autonomia diz respeito ao nível de interferência do ambiente de aprendizagem na postura dos envolvidos e nas etapas do processo de aprendizagem. Quanto maior a autonomia, maior a liberdade que os aprendizes possuem de conduzir seu próprio processo de aprendizagem, definindo metodologias e critérios próprios para alcançar os objetivos [Bragança et.al., 2008].

Os projetos de aprendizagem (PAs) no ambiente escolar permitem que conceitos e sistemas conceituais dos aprendizes sejam ativados na formulação de questões de investigação de modo autônomo, visando a aproximação entre o ambiente escolar formal e o ambiente de vivência e experiência dos alunos [Fagundes et al., 2006]. Dessa forma, os temas para os PAs são de livre negociação, partindo-se das experiências prévias e dos interesses de cada aprendiz [Tavares et al., 2001].

\section{Metodologia dos projetos de aprendizagem}

Os projetos de aprendizagem são desenvolvidos conforme etapas sistemáticas previamente definidas pela arquitetura [Fagundes et al., 2006]. As etapas são formadas por criações e registros de conteúdos com autoria dos aprendizes, já que essa arquitetura coloca os próprios estudantes como sujeitos ativos no desenvolvimento dos projetos [Carvalho et al., 2005].

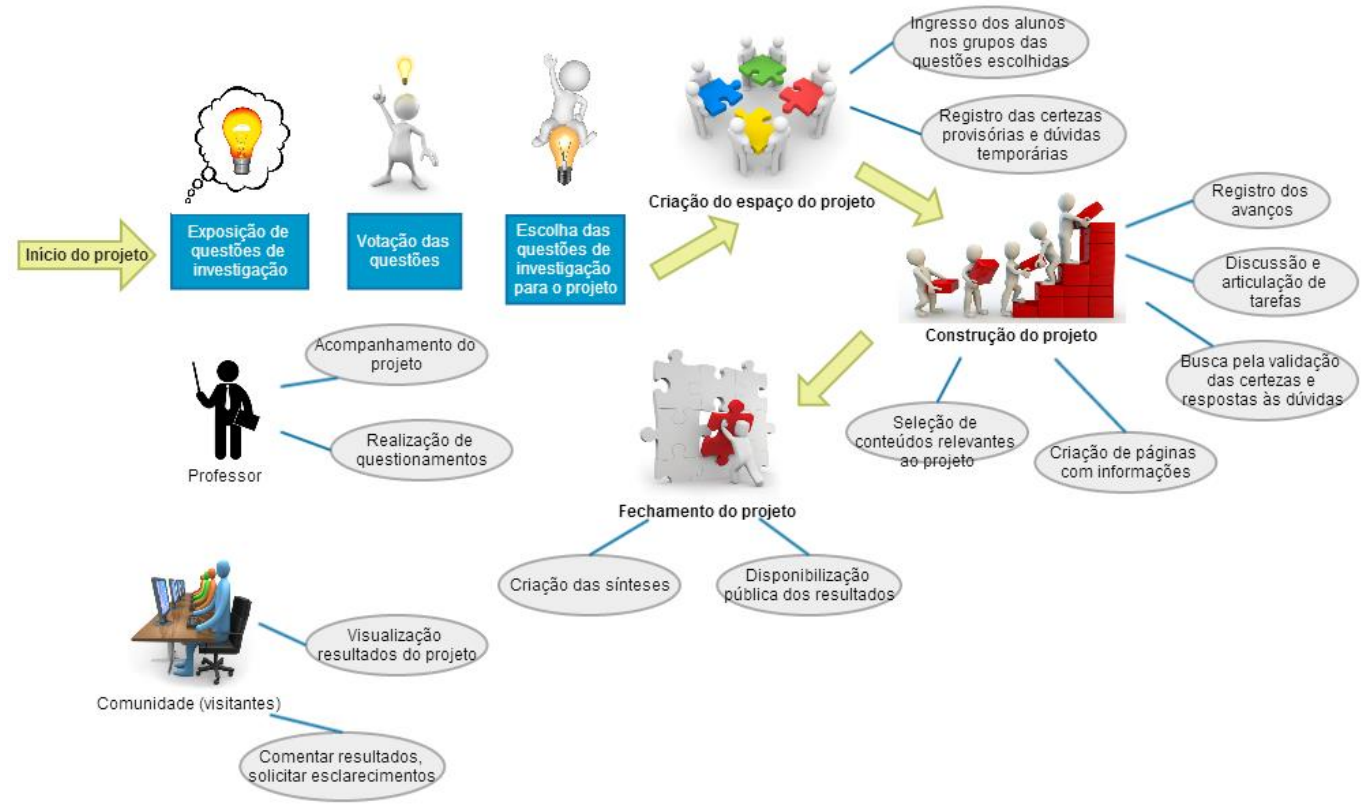

Figura 1. Processo de desenvolvimento dos projetos de aprendizagem

Os PAs são iniciados com o levantamento de questões presentes na vivência do aluno e que o instigam a buscar soluções para elas. Após isso, é realizado uma etapa de agrupamento dos estudantes em torno das questões de investigação até que todos os estudantes estejam alocados em um grupo com a respectiva questão de investigação. A seguir, cada grupo de estudantes inicia uma etapa de levantamento das certezas provisórias (“o que sei”) e das dúvidas temporárias ("o 
que preciso saber") sobre cada questão de investigação. Esse levantamento é também chamado de inventário do conhecimento prévio e norteia todas as ações no processo de cada PA [Mergendoller et.al., 2000].

Em outro momento, os alunos se articulam para filtrar as certezas e dúvidas que são relevantes ao PA. A contextualização desse levantamento inicial forma as unidades de investigação e é por meio delas que são realizadas as pesquisas, as entrevistas e as consultas que visam refutar, validar e responder as dúvidas e certezas dentro das unidades de investigação. A cada momento, elas podem ser acrescidas de novas certezas e dúvidas e assim o inventário é modificado [Fagundes et al., 2006].

À medida em que todas as unidades de investigação vão sendo populadas com conteúdos, os alunos elaboram relatórios apresentando as fontes, os procedimentos e as discussões que levam àqueles resultados, bem como produzem mapas conceituais que permitem a visualização dos conhecimentos levantados no PA. Assim que ocorre o fechamento do projeto, os alunos desenvolvem as sínteses finais que respondem e solucionam a questão de investigação e publicam materiais de apresentação para serem apreciados pela comunidade.

A apresentação e publicação dos resultados constituem a etapa final do PA e permite um feedback da comunidade que possa levar ao repensar de algum ponto do projeto. A socialização dos projetos também é um fator importante, quando alunos envolvidos em outras questões de investigação têm acesso aos resultados e fazem comentários e críticas que possam fornecer possíveis pontos de ampliação do projeto ou ligação com os demais PAs [Barron et al., 1998].

Durante todas as etapas o professor exerce a função de orientar os alunos no projeto, articular, incentivar, motivar, solicitar esclarecimentos, questionar o que está sendo feito, bem como avaliar os progressos visualizando os diários de bordos e as atividades desenvolvidas, intercedendo sempre que julgar necessário.

\section{Ambientes de suporte a projetos de aprendizagem}

O desenvolvimento de PAs necessita de ferramentas digitais específicas, principalmente para a mediação das interações não presenciais internas aos grupos de aprendizes [Fagundes et al., 2006]. Durante o desenvolvimento dos projetos, os professores têm múltiplos papéis, como o incentivo e acompanhamento dos aprendizes, coordenação das atividades desenvolvidas em cada projeto etc. Já os alunos são os agentes ativos na construção da rede de conhecimento dentro do projeto e os próprios articuladores das ações e do respectivo grupo.

Para apoiar a realização dos PAs, alguns ambientes foram concebidos, propondo-se a mediar o processo necessário ao desenvolvimento das etapas dos PAs, conforme apresentam as seções 3.1 e 3.2 .

\subsection{AMADIS}

O AMADIS é um ambiente de suporte a quatro atividades pedagógicas: desenvolvimento de projetos de aprendizagem, oficinas tecnológicas, seminários temáticos e formação de grupos de interesse [Fagundes et al., 2006]. 
Quanto aos projetos de aprendizagem, o sistema gerencia a criação de novos projetos, os documentos criados e permite a navegação nesses projetos. O sistema conta com ferramentas básicas de comunicação como chat, fórum, mural e email e também ferramentas de apoio individual como diário pessoal e página pessoal.

\subsection{Ambiente de Apoio ao Desenvolvimento de Projetos de Aprendizagem (ADEPA)}

O segundo ambiente de apoio ao desenvolvimento de projetos de aprendizagem é o ADEPA. Ele se baseia no AMADIS para oferecer um ambiente de desenvolvimento de projetos de aprendizagem com a integração dos vários atores envolvidos (professores, alunos, pais e visitantes) [Monteiro, 2006].

Esse ambiente possui 3 módulos para a comunicação e interação no ambiente: controle de usuários, controle de projetos, acompanhamento e avaliação dos projetos.

Para inserção de conteúdos, o ambiente possui a classe "Artigos", onde uma vez inserido o conteúdo, ele é classificado em certeza, dúvida, diário de bordo etc.

\section{Limitações dos ambientes atuais}

Dentre as limitações apresentadas pelos ambientes de projetos de aprendizagem atuais, destacase a abrangência limitada dos mesmos, fazendo com que haja necessidade de utilização de ferramentas externas (hospedadas na Internet ou programas de terceiros) para apoio à construção de conteúdos referentes às etapas dos projetos de aprendizagem (levantamento das questões de investigação, seleção das questões mais interessantes para a turma, formação dos grupos de aprendizes associados à respectiva questão de investigação, registro de certezas provisórias e dúvidas temporárias, criação de unidades de investigação, elaboração e publicação de mapas conceituais, elaboração em grupo e publicação de sínteses, etc). No modus operandi destes ambientes, os conteúdos são gerados externamente por meios de ferramentas e os resultados são exportados em documentos e enviados (upload) ao ambiente. Como consequência dessa prática, os conteúdos ficam estacionados em repositórios, sem permitirem qualquer tipo de integração e manipulação, o que dificulta a construção do conhecimento. Além disso, a carência de suporte às etapas dos projetos de aprendizagem desfavorecem a produção de conteúdo contextualizada pois não consideram o momento (etapa) em que o conteúdo está sendo produzido e nem com o formato do mesmo. Desse modo, não é possível acompanhar a evolução dos conteúdos e consequente evolução da construção do conhecimento dos aprendizes, o que afeta negativamente o desenvolvimento dos projetos de aprendizagem, como explicita Fagundes et al. (2006) "[...] todas as micro-atividades de um projeto usam e produzem informações que necessitam ser interligadas, em forma de rede, para que os seus desenvolvedores possam a qualquer instante navegar por ele, revendo lições aprendidas, revisando decisões tomadas, reaproveitando informações etc $[\ldots]$ ".

A carência de mecanismos de log (histórico das ações ao longo do percurso realizado) e acompanhamento das ações de autoria de conteúdos é uma outra deficiência dos ambientes atuais e prejudica diretamente o professor. Como não há uma forma integrada e eficiente de observar o 
progresso tanto dos indivíduos quanto dos conteúdos, o professor acaba por ser obrigado a navegar por todas as ferramentas externas (sites, softwares) utilizadas por cada etapa do projeto para verificar a construção do conteúdo e participação dos aprendizes, o que é muito custoso e também ineficiente pois é inconcebível acompanhar vários projetos com várias questões de investigação e muitos aprendizes desta forma dispersa e informal de acompanhamento, refletindo no poder de avaliação, articulação e participação do professor no projeto.

\section{Proposta do Ambiente para Projetos de Aprendizagem}

Um ambiente propício ao desenvolvimento de projetos de aprendizagem precisa ser flexível para que os projetos de aprendizagem sejam conduzidos de acordo com as necessidades, experiências e nível de conhecimento dos aprendizes. Não parece ser interessante um ambiente sistemático seguindo uma estrutura de "next-next-finish" que conduz as etapas e solicita aos aprendizes a inserção de conteúdos independente dos anseios dos participantes do projeto. O ambiente deve sim oferecer mecanismos e ferramentas capazes de compreender cada etapa do projeto, mas ele não pode interferir no poder de articulação dos envolvidos, levando-os à adoção de práticas diferentes daquelas combinadas entre os próprios aprendizes e o professor. $\mathrm{O}$ ambiente deve preservar a autonomia dos aprendizes diante dos problemas e possibilitar a utilização de ferramentas já compreendidas por eles e difundidas na cultura Web 2.0 como páginas colaborativas, discussão e blogging, bem como ferramentas específicas de apoio às etapas aos projetos de aprendizagem (como criação de unidades de investigação, por exemplo) em prol do desenvolvimento do projeto e consequente elucidação da questão de investigação.

Além disso, o ambiente precisa inserir o professor em um contexto que ele possa participar do projeto seguindo seus múltiplos papéis de questionador, motivador, articulador e observador oferecendo mecanismos eficientes de acompanhamento e participação nos projetos de aprendizagem.

\subsection{Metodologia}

A especificação de um ambiente propício para suporte a projetos de aprendizagem surgiu com base em análises de desenvolvimento de projetos de aprendizagem à distância pela Internet (possibilitada por ferramentas de propósito geral presentes na grande rede como Wikis, batepapos e fóruns) e em um segundo momento, da análise dos ambientes que se propõem a apoiar o desenvolvimento de projetos de aprendizagem. Desta forma, foi possível identificar as falhas dos ambientes e das ferramentas atuais em apoiarem as etapas dos projetos.

Devido ao grande fluxo de interações que envolvem as etapas dos projetos de aprendizagem, houve a adoção da metodologia de engenharia de software em cascata, onde cada ferramenta foi concebida individualmente desde a etapa inicial de levantamento dos requisitos até a implantação (agregação) da ferramenta ao núcleo. A metodologia de desenvolvimento seguida encontra-se detalhada na figura a seguir. 


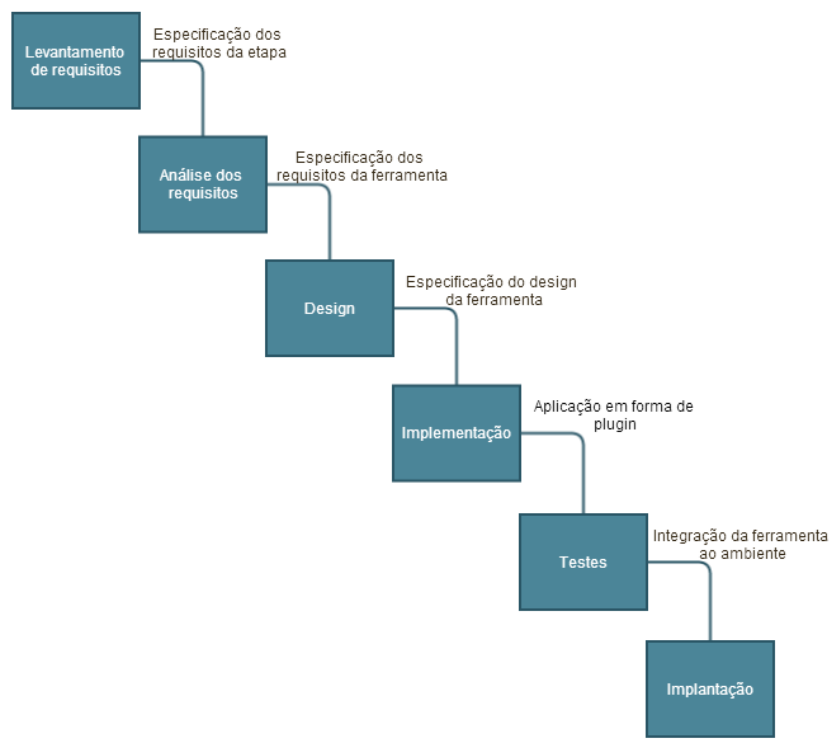

Figura 2. Modelo de desenvolvimento das ferramentas de suporte às etapas de projetos de aprendizagem

\subsection{Ambiente para Projetos de Aprendizagem}

O Ambiente para Projetos de Aprendizagem (AProAp) foi construído pelos autores para apoiar o desenvolvimento de PAs. Ele é um ambiente integrado, com ferramentas direcionadas para o suporte a cada etapa de um PA, bem como de mecanismos de acompanhamento das atividades e conteúdos.

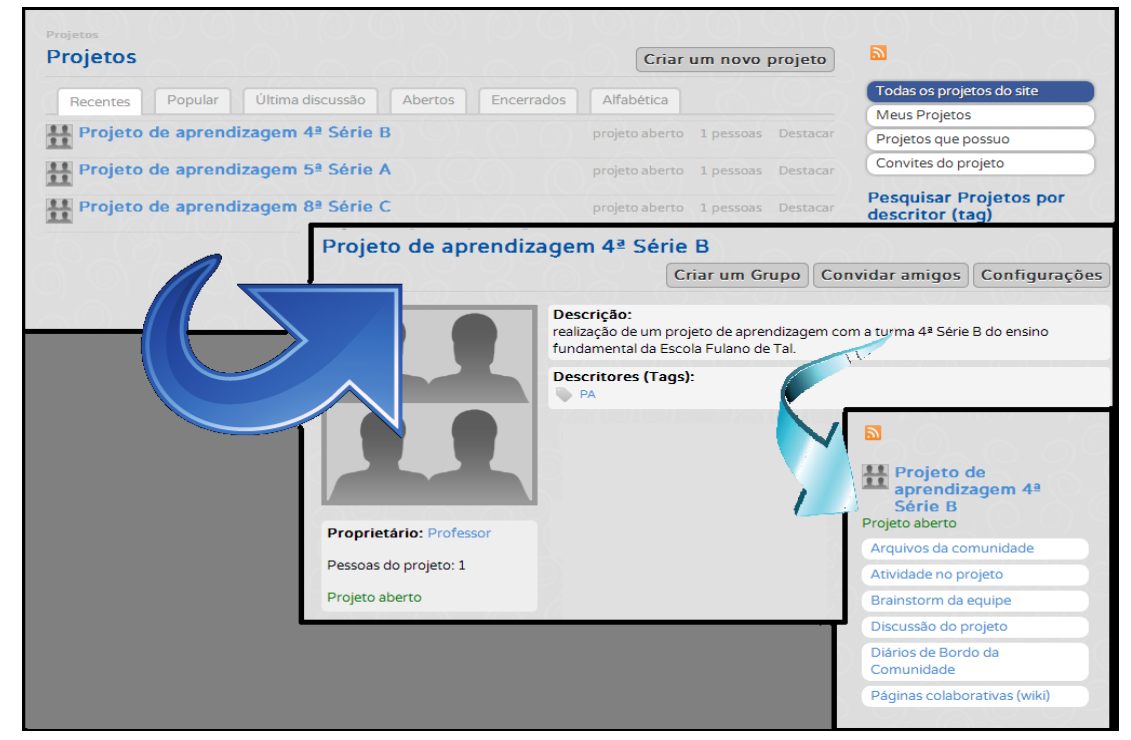

Figura 3. Visão conceitual do AProAp

Por meio do AProAp, é possível a realização de todas as etapas que compõem os PAs, desde as etapas de preparação para os PAs (levantamento das questões de investigação e formação dos grupos de desenvolvimento de PAs, com as respectivas questões de investigação) até as etapas finais dos mesmos (criação e publicação de conteúdos). Desse modo, o professor 
pode acompanhar todos os projetos (visualizando mudanças de conteúdos e registro de atividades), mediar debates, trocar de mensagens para tirar dúvidas e acompanhar as etapas dos projetos. Os aprendizes são capazes de ingressarem em projetos, criarem grupos para questões de investigação e unidades de investigação e assim usarem as diversas ferramentas de suporte às etapas do PA.

Cada ferramenta do ambiente está associada a uma etapa dos PAs (Figura 5) e gera conteúdos que ficam disponíveis para o uso nas etapas subsequentes, cada uma delas contendo um mecanismo de acompanhamento (coletivo, individualizado) e algumas delas com mecanismos de versionamento, permitindo o acompanhamento da evolução do conteúdo, ao longo das modificações. A Figura 5 mostra as etapas de um projeto de aprendizagem e as ferramentas disponíveis no AProAp para cada etapa.

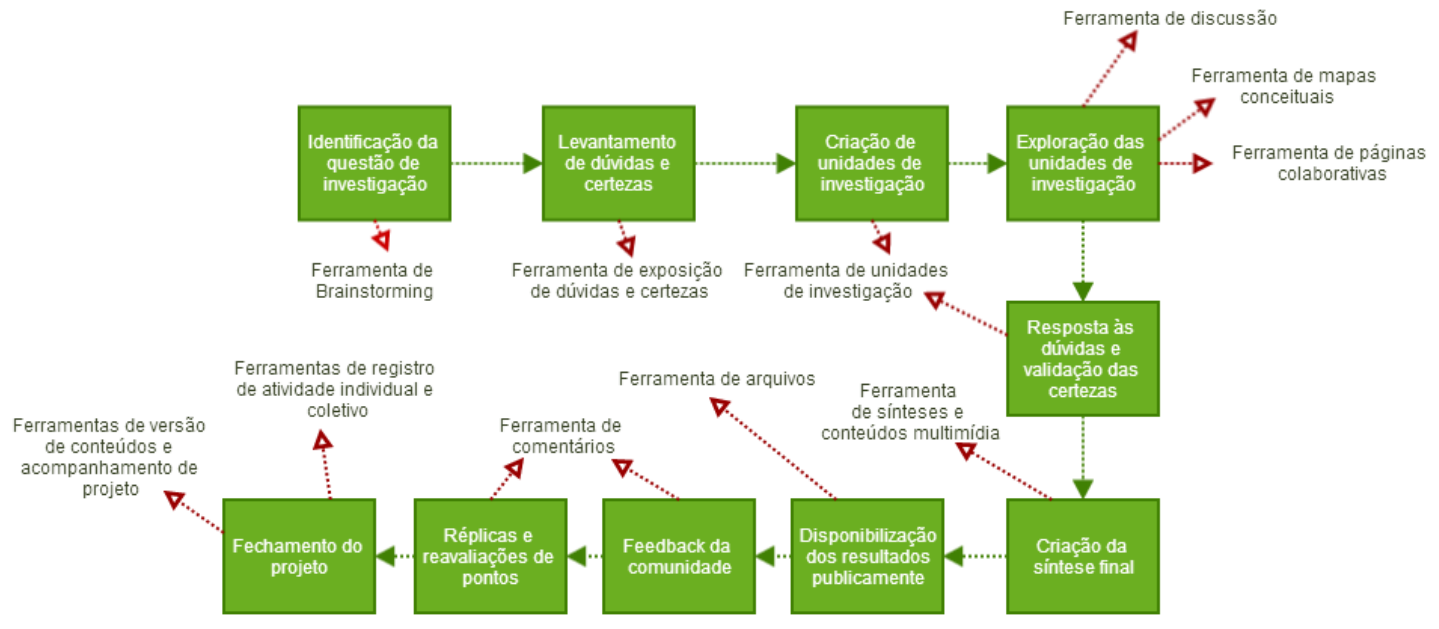

Figura 4. Ferramentas necessárias às etapas dos projetos de aprendizagem

Em cada etapa do PA há ferramentas que ajudam os aprendizes a registrarem seus avanços e a construírem conteúdos de maneira autônoma. Algumas ferramentas também oferecem ao professor meios de acompanhar as atividades desenvolvidas e permitem a mediação necessária, tais como:

- Questões de investigação: é uma ferramenta voltada para exposição, votação e escolha de questões de investigação. No início dos PAs é preciso que se permita a livre exposição de questões por parte dos aprendizes, buscando levantar questões de investigação de interesse para eles. Em um segundo momento, é preciso identificar as questões mais relevantes à turma para que grupos de interesse sejam formados em torno dessas questões mais relevantes. No terceiro momento, é preciso que sejam explicitadas as questões de interesse da turma formar grupos, segundo os interesses individuais pelas questões. A ferramenta é projetada para dar suporte a esses três momentos importantes da etapa "identificação da questão de investigação".

- Discussão: a comunicação é essencial dentro dos PAs, seja juntamente com o professor, para momentos de orientação e definição de objetivos e métodos, seja entre os aprendizes na identificação e validação de conteúdos que farão parte do projeto. A ferramenta possibilita o debate entre os participantes do PA e é usada em todas as etapas do projeto.

- Registro de certezas provisórias e dúvidas temporárias: é uma ferramenta que coleta do grupo tanto as certezas provisórias quanto as dúvidas temporárias sobre a questão de 
investigação. Essa ferramenta oferece um espaço coletivo para livre exposição e organização desse conhecimento prévio pelo grupo de cada questão de investigação.

- Unidades de investigação: a etapa de seleção do conhecimento prévio prevê o agrupamento das certezas provisórias e das dúvidas temporárias mais relevantes para a questão de investigação do PA. O caráter da etapa é cíclico, o que significa que haverá alterações de conteúdos dessas unidades com o acréscimo de novas certezas e dúvidas, além de informações de validação das certezas e de respostas às dúvidas, ao longo de todo o projeto. A ferramenta de unidades de investigação permite a criação de páginas públicas com atualizações constantes das dúvidas temporárias e das certezas provisórias que compõem uma unidade de investigação. À medida que são conseguidas novas informações para validar as certezas e responder às dúvidas, ocorre o versionamento das páginas, para permitir acompanhar as alterações realizadas em cada página, com as respectivas autorias.

- Diário de bordo: durante todas as etapas dos PAs há o registro individual ou coletivo realizado pelos envolvidos no projeto. A ferramenta "diário de bordo" preenche essa necessidade de coletar relatos dos aprendizes durante o projeto. Para possibilitar ainda mais a livre exposição, a ferramenta permite que seja definido o público de cada conteúdo, que pode ser privado (apenas para o indivíduo ou grupo) ou público.

- Editor de mapas conceituais: esta ferramenta permite a construção de mapas conceituais, de modo a permitir a representação gráfica do conhecimento que é levantado durante a realização de cada PA.

- Organizador de grupos: os PAs incluem trabalhos coletivos dos aprendizes, que são agrupados de acordo com seus interesses nas questões de investigação. Todas as tarefas de agrupamento e gerenciamento dos grupos são facilitadas por essa ferramenta que permite a criação de projetos, permissões de conteúdos, aprovação de membros, configuração de ferramentas do projeto e repositório dos conteúdos.

- Ferramentas de acompanhamento individual e coletivo de projetos: permitem que o professor acompanhe múltiplos projetos, com dados qualitativos e quantitativos individuais ou coletivos dos autores dos projetos. O AProAp se concentra nos mecanismos de acompanhamento do professor tanto quanto no suporte ao aprendiz nas etapas dos projetos.

Além das ferramentas, o AProAp possui mecanismos de visualização e visitas por parte da comunidade virtual. Pessoas não envolvidas com os projetos podem ter acesso ao ambiente e aos resultados dos projetos, para realizarem questionamentos ou observações sobre o conteúdo, a serem esclarecidos pelos autores de cada projeto. A política de privacidade do ambiente é definida pelo administrador e a privacidade dos conteúdos é definida pelos próprios autores.

\section{Resultados}

O AProAp atualmente está na etapa de validação, em disciplinas oferecidas pelo Programa de Pós-Graduação (PPGI) da UFES. Os resultados iniciais sugerem que o ambiente facilita o desenvolvimento dos PAs e permite suporte importante aos grupos e ao professor durante as etapas de desenvolvimento dos projetos de aprendizagem. Para efeitos de validação por parte da comunidade livre, também foram disponibilizadas duas das ferramentas de apoio a projetos de aprendizagem presentes no AProAp para download em repositório público da Internet 
(ferramenta de questões de investigação ${ }^{1}$ e de registro individual de atividades ${ }^{2}$ ). Os feedbacks advindos de usuários que obtiveram as ferramentas foram positivos, com elogios à iniciativa e à utilidade das ferramentas. $\mathrm{O}$ interesse da comunidade digital por essas ferramentas pode ser confirmado pelos mais de 90 downloads realizados até o presente momento.

\section{Conclusão e trabalhos futuros}

Os ambientes atuais que se propõem a dar suporte à pedagogia de projetos, em específico aos projetos de aprendizagem, falham em oferecer suporte para as etapas dos projetos, seja pela ausência de ferramentas integradas, seja pela ausência de foco no desenvolvimento do projeto. Por isso, o Ambiente para Projetos de Aprendizagem (AProAp) foi projetado para oferecer ferramentas e espaços dedicados ao desenvolvimento dos projetos de aprendizagem, desde a preparação para o projeto até a publicação dos resultados para a comunidade. Diferentemente dos demais ambientes, o AProAp foi construído para acompanhar a evolução do conteúdo e do projeto em si, por meio de recursos de versionamento e acompanhamento, não se limitando a ser um repositório de conteúdos consolidados.

Como trabalho futuro, pretende-se povoar o ambiente com agentes sintéticos baseados em conhecimento, capazes de auxiliar o aprendiz nas etapas dos projetos de aprendizagem, ajudá-lo a refletir sobre o conhecimento levantado por ele e pelos colegas do mesmo grupo e de outros grupos, além de agentes que o ajudem a encontrar textos relevantes e informações sensíveis ao contexto.

\section{Referências}

Barron, B. JS et al. (1998) "Doing with understanding: Lessons from research on problem-and project-based learning". Journal of the Learning Sciences, v. 7, n. 3-4, p. 271-311.

Bragança, B; Augusto, L.; Pontelo, I. (2008) "Práticas educativas e ambientes de aprendizagem escolar: relato de três experiências". I Seminário Nacional de Educação Profissional e Tecnológica. Belo Horizonte.

Carvalho, M.J. S.; Nevado, R.A., Menezes, C.S. (2005) "Arquiteturas Pedagógicas para Educação à Distância: Concepções e Apoio Telemático". Anais do Simpósio Brasileiro de Informática na Educação. v. 1, n.1, p. 351-360.

Fagundes, L. C., et al. (2005) "AMADIS-Um Ambiente Virtual para apoio ao Desenvolvimento de Projetos de Aprendizagem". Anais do Simpósio Brasileiro de Informática na Educação. v. 1, n. 1, p.298-308.

Fagundes, L.C., et al. (2006) "Projetos de Aprendizagem - uma experiência mediada por ambientes telemáticos". Revista brasileira de informática na educação. v. 14, n. 1, p. 29-39.

Mergendoller, J. R.; Thomas, J. W. (2000) "Managing project based learning: Principles from the field". Annual Meeting of the American Educational Research Association, New Orleans.

Monteiro, V. C. P. C. (2006) "Um ambiente de apoio ao desenvolvimento de Projetos de Aprendizagem”. Dissertação de Mestrado, PPGI-UFES, Vitória-ES.

${ }^{1}$ Disponível em http://goo.gl/6OhLwo

${ }^{2}$ Disponível em http://goo.gl/LcRh5q 
III Congresso Brasileiro de Informática na Educação (CBIE 2014)

XXV Simpósio Brasileiro de Informática na Educação (SBIE 2014)

Ríos, I. de los; et al. (2010) "Project-based learning in engineering higher education: two decades of teaching competences in real environments". Procedia-Social and Behavioral Sciences, v. 2, n. 2, p. 1368-1378.

Tavares, O. L; Brito, S. R.; Souza, R. S. ; Menezes, C. S. (2001) "Ambiente de apoio à mediação de aprendizagem: uma abordagem orientada por processos e projetos". Revista Brasileira de Informática na Educação, p. 77-87. 